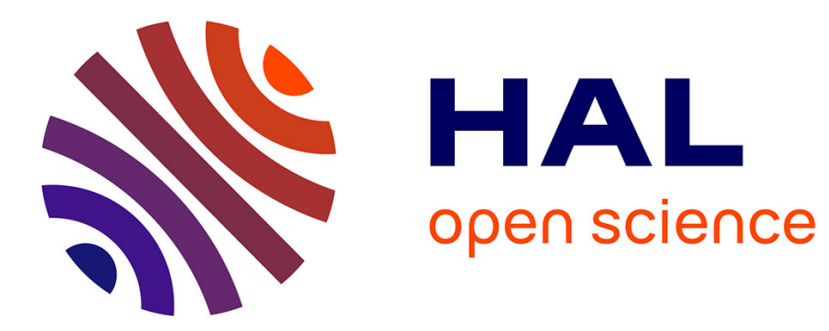

\title{
Pitch body orientation influences the perception of self-motion direction induced by optic flow
}

\author{
Aurore Bourrelly, J. -L. Vercher, L. Bringoux
}

\section{To cite this version:}

Aurore Bourrelly, J. -L. Vercher, L. Bringoux. Pitch body orientation influences the perception of self-motion direction induced by optic flow. Neuroscience Letters, 2010, 482 (3), pp.193-197. 10.1016/j.neulet.2010.07.028 . hal-01436028

\section{HAL Id: hal-01436028 \\ https://hal.science/hal-01436028}

Submitted on 2 May 2018

HAL is a multi-disciplinary open access archive for the deposit and dissemination of scientific research documents, whether they are published or not. The documents may come from teaching and research institutions in France or abroad, or from public or private research centers.
L'archive ouverte pluridisciplinaire HAL, est destinée au dépôt et à la diffusion de documents scientifiques de niveau recherche, publiés ou non, émanant des établissements d'enseignement et de recherche français ou étrangers, des laboratoires publics ou privés. 
Provided for non-commercial research and education use. Not for reproduction, distribution or commercial use.

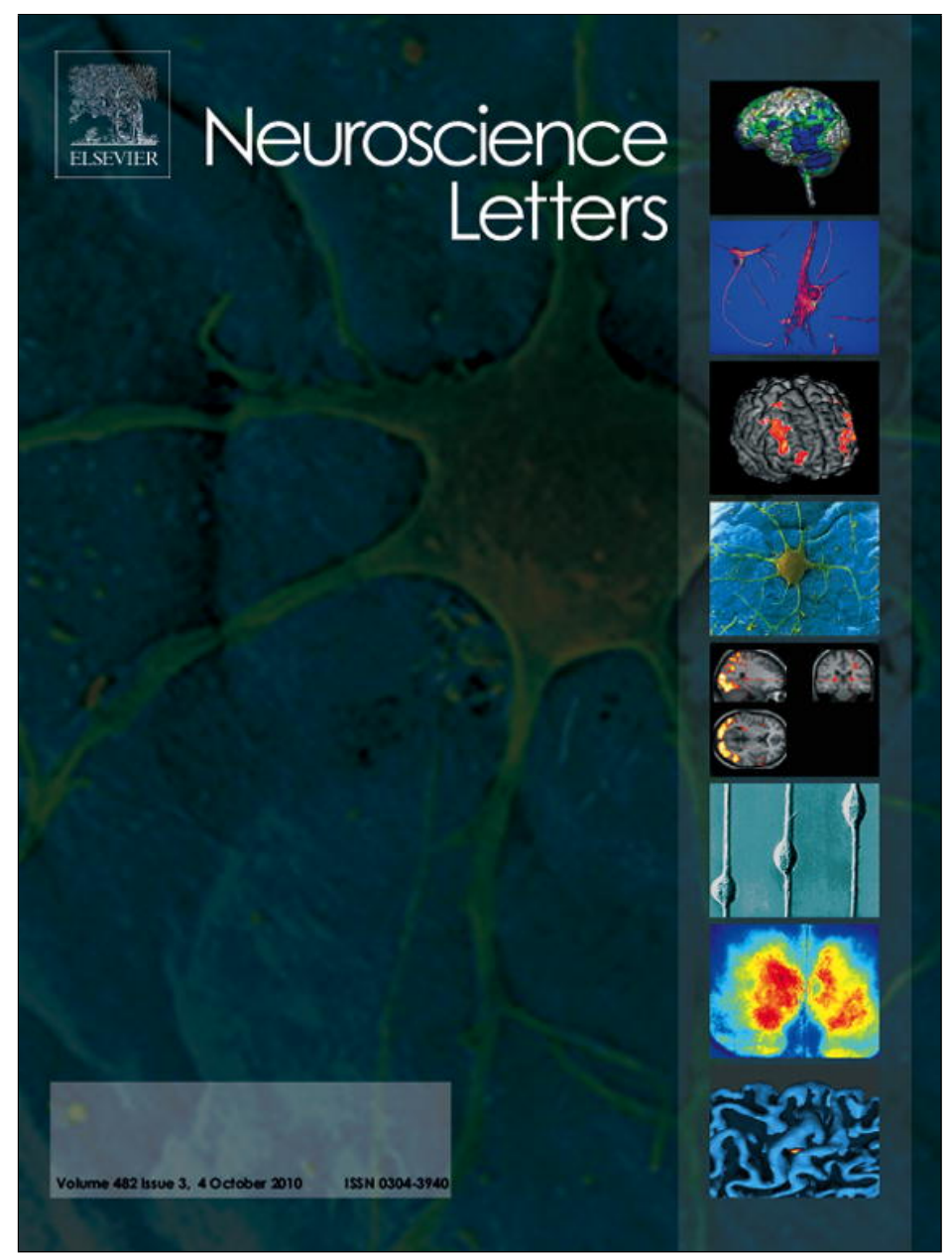

This article appeared in a journal published by Elsevier. The attached copy is furnished to the author for internal non-commercial research and education use, including for instruction at the authors institution and sharing with colleagues.

Other uses, including reproduction and distribution, or selling or licensing copies, or posting to personal, institutional or third party websites are prohibited.

In most cases authors are permitted to post their version of the article (e.g. in Word or Tex form) to their personal website or institutional repository. Authors requiring further information regarding Elsevier's archiving and manuscript policies are encouraged to visit:

http://www.elsevier.com/copyright 


\title{
Pitch body orientation influences the perception of self-motion direction induced by optic flow
}

\author{
A. Bourrelly*, J.-L. Vercher, L. Bringoux \\ UMR CNRS 6233, 'Institut des Sciences du Mouvement Etienne-Jules Marey', CNRS E' Aix-Marseille Université, 163, avenue de Luminy, CP 910, 13288 Marseille, France
}

\section{A R T I C L E I N F O}

\section{Article history:}

Received 3 March 2010

Received in revised form 13 July 2010

Accepted 13 July 2010

\section{Keywords:}

Spatial perception

Body orientation

Heading

Self-motion

Reference frame

Geocentric

Egocentric

\begin{abstract}
A B S T R A C T
We studied the effect of static pitch body tilts on the perception of self-motion direction induced by a visual stimulus. Subjects were seated in front of a screen on which was projected a 3D cluster of moving dots visually simulating a forward motion of the observer with upward or downward directional biases (relative to a true earth horizontal direction). The subjects were tilted at various angles relative to gravity and were asked to estimate the direction of the perceived motion (nose-up, as during take-off or nosedown, as during landing). The data showed that body orientation proportionally affected the amount of error in the reported perceived direction (by $40 \%$ of body tilt magnitude in a range of $\pm 20^{\circ}$ ) and these errors were systematically recorded in the direction of body tilt. As a consequence, a same visual stimulus was differently interpreted depending on body orientation. While the subjects were required to perform the task in a geocentric reference frame (i.e., relative to a gravity-related direction), they were obviously influenced by egocentric references. These results suggest that the perception of self-motion is not elaborated within an exclusive reference frame (either egocentric or geocentric) but rather results from the combined influence of both.
\end{abstract}

(c) 2010 Elsevier Ireland Ltd. All rights reserved.
When one moves in a visual environment, the pattern of optic flow projected on the retina gives information about the direction of our self-motion. By recreating this visual pattern, a feeling of selfmotion can be fully experienced by static observers on the sole basis of the visual context $[8,10,26]$. Hence, investigating self-motion induced by an optic flow field is of interest for questioning the perceived displacement of operators embarked in a vehicle $[8,10]$. Specifically, in the context of aeronautics, the way pilots perceive the up and down direction of self-motion from optic flow is critical for flight safety $[9,10]$. For instance, visual angle accuracy on the order of $\pm 1^{\circ}$ was shown to be required for the control of visual self-motion and obstacle avoidance $[9,27]$.

For more than 50 years, behavioural neuroscientists have mainly raised the question of self-motion perception involving egocentric or allocentric tasks which respectively required to be assessed relative to body-fixed elements or other features of the visual environment. For instance, subjects were usually asked to point a cursor toward the perceived direction of self-motion on the visual scene relative to their straight ahead $[26,28]$, or conversely had to discriminate the direction of self-motion as oriented leftward or rightward relative to a stationary post in the environment $[8,27,28]$. Overall, these judgments were shown to be accurately performed,

\footnotetext{
* Corresponding author. Tel.: +33 4911722 62; fax: +33 491172252. E-mail addresses: aurore.bourrelly@hotmail.fr (A. Bourrelly), lionel.bringoux@univmed.fr (L. Bringoux).
}

mainly on the basis of the optic flow pattern. However, only a few studies investigated the perception of self-motion direction through a task, which must be referred to some earth-invariant directions, such as the direction of the gravity or the horizon. This may be of particular importance in a context of poor visibility (during night or foggy days) when relevant visual-polarity cues for up and down directions are no more available. Within this context, judging upward and downward direction of self-motion represents a geocentric task which is required to be achieved relative to an earth-based reference. In this particular case, body orientation can be dissociated from the direction of self-motion relative to gravity. Specifically, we hypothesize that the relative reference for upward and downward judgments may be the subjective horizon that is the perceived plane passing through the eye and normal to gravity.

Several studies previously focused on the perception of the subjective horizon at different body orientations [4,5]. It was shown that static pitch body tilts influenced the perceptual estimates of the horizon. In line with these previous findings, the present experiment was designed to investigate whether pitch body orientation can modify the perceived direction of a forward self-motion induced by optic flow, with upward and downward directional biases relative to a true earth horizontal direction. Specifically, since body orientation was previously found to exert an influence on earth-based judgments, it was hypothesized that the perception of self-motion direction induced by optic flow can be also affected by static body tilt when the judgment must be referred to external space. 


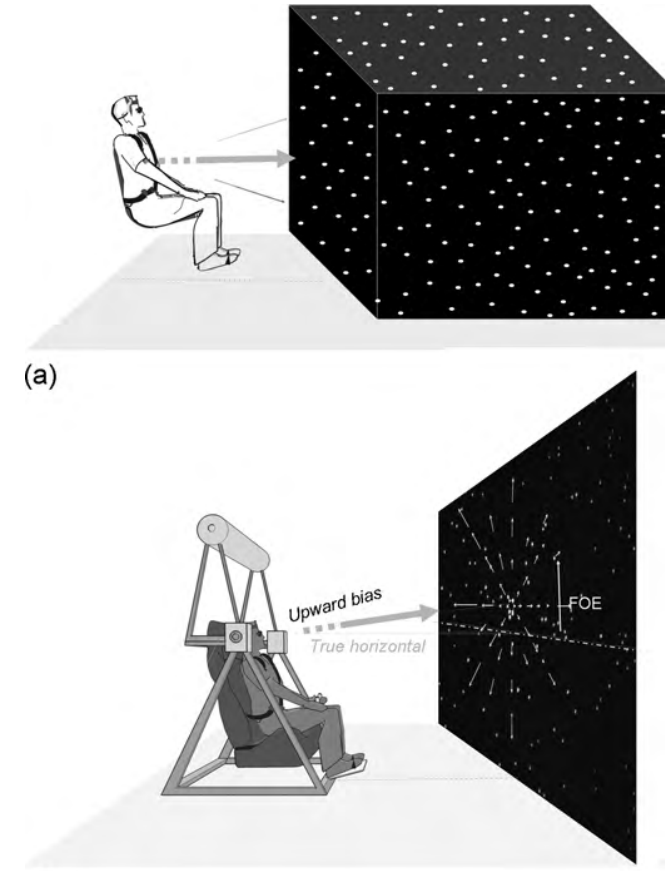

(c)

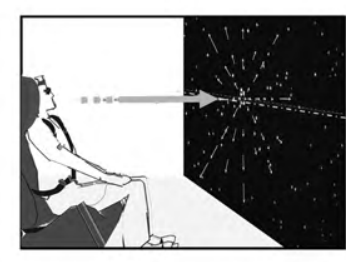

(b)

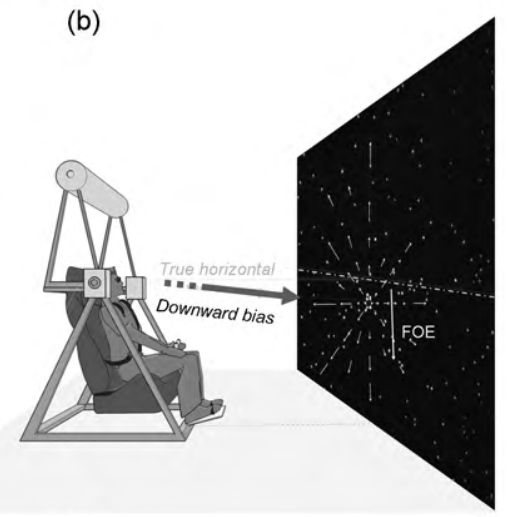

(d)

Fig. 1. (a) Illustration of the optic flow display presented in a 3D virtual environment. The visual stimulus simulated an optic flow field viewed by an observer translating forward into a 3D cloud of stationary dots. (b) Relative to the stationary observer, the projected stimulus consisted in a cluster of 400 circular dots which radially expanded toward him. Upward (c) or downward (d) directional biases relative to a true earth horizontal direction were inserted in the simulated forward motion by shifting on the screen the elevation of the central focus of expansion of the moving cloud of dots along the subjects' median plane. The observer experimented a feeling of self-motion in a direction that was tilted either upward (c) or downward (d) relative to the earth horizontal direction.

Twelve subjects ( 5 males and 7 females; mean age $26.5 \pm 6.67$ ) with normal or corrected-to-normal vision (by lens correction), gave their informed consent to participate in the study, in compliance with the ethical committee regulating human experimentation in France. They had no previous history of vestibular and neurological symptoms. All were naive as to the hypothesis under study.

Subjects were seated on a padded tilting chair allowing body rotations in pitch with acceleration above the vestibular threshold for rotation perception $[1,11]$. The chair was rotated at constant velocity during $11 \mathrm{~s}$, with a period of initial acceleration and final deceleration of $2 \mathrm{~s} ;\left(0.4^{\circ} \mathrm{s}^{-1}\right.$ and $0.2^{\circ} \mathrm{s}^{-2}$ for $\pm 5^{\circ}$ tilt, $0.8^{\circ} \mathrm{s}^{-1}$ and $0.4^{\circ} \mathrm{s}^{-2}$ for $\pm 10^{\circ}$ tilt, $1.2^{\circ} \mathrm{s}^{-1}$ and $0.6^{\circ} \mathrm{s}^{-2}$ for $\pm 15^{\circ}$ tilt, $1.6^{\circ} \mathrm{s}^{-1}$ and $0.8^{\circ} \mathrm{s}^{-2}$ for $\pm 20^{\circ}$ tilt). They were restrained by means of a shoulder harness with their head strapped and secured on a headrest so that the naso-occipital axis was orthogonal to the direction of the gravity when the chair was vertically oriented. The axis of rotation of the chair coincided with the trans-ocular axis. In this way, the subjects' eye level was kept at the same height with respect to the floor reference $(1.34 \mathrm{~m})$ regardless of the tilt magnitude. Subjects were placed in front of a screen which subtended an elliptic visual angle of $81^{\circ}$ horizontal $\times 42^{\circ}$ vertical when viewed binocularly by observers wearing customised goggle at a distance of $1.22 \mathrm{~m}$. This ensured that the squared edges of the screen were masked. The visual stimulus was generated by a PC Dell Precision 380 computer via our in-house ICE software and projected onto the screen by a video-projector (refresh rate set to 85 frames/s). The visual stimulus was set to simulate an optic flow field viewed by an observer translating forward into a 3D cloud of stationary dots (Fig. 1a). Relative to the stationary observer, the projected stimulus consisted in a cluster of 400 circular dots (diameter $=5 \mathrm{~mm}$ without local expansion) which radially expanded toward him (Fig. 1b). Upward or downward directional biases relative to a true earth horizontal direction were inserted in the simulated forward motion by shifting on the screen the elevation of the central focus of expansion of the mov- ing cloud of dots along the subjects' median plane (Fig. 1c and d). In this way, the observer experienced a feeling of self-motion in a direction that was tilted either upward or downward relative to the earth horizontal direction (as during an aircraft take-off or landing, respectively). The velocity flow field was designed to simulate a constant speed of $66 \mathrm{~m} \mathrm{~s}^{-1}$ in order to be close to the actual condition of flight during aircraft landing. The total number of dots was always kept constant on the screen so that new dots appeared at randomly determined positions in the screen when others went out.

Subjects were first positioned at the desired body angle relative to gravity after $15 \mathrm{~s}$ of rotation in complete darkness, followed by $15 \mathrm{~s}$ of rest. This specific duration was chosen as a compromise between the weakest vestibular resting discharge allowing to consider post-rotational effects as negligible and limited somatosensory adaptation due to the subsequent period of static tilt $[1,11]$. Stationary subjects were then asked to open their eyes and to observe the visual stimulus. The visual stimulus was presented motionless for $0.5 \mathrm{~s}$ before simulating self-motion for $2 \mathrm{~s}$. During this phase, subjects were clearly required to keep the eye still in the direction in which they felt translating. Next, subjects were asked to respond to the following question: "Do I feel translating upward relative to the earth horizontal direction?" or "Do I feel translating downward relative to the earth horizontal direction?" They were asked to give their response at the end of the visual presentation after the scene disappeared, respectively with a right or a left hand-held button. Judgment settings were recorded via the ADwin-Pro system (Keithley ${ }^{\odot}$ ) piloted via our in-house Docometer software. At the end of the sequence, the chair was brought back to the vertical and the room lights were turned on for $5 \mathrm{~s}$ before a new sequence was launched.

Nine body orientations $\left(0^{\circ} ;-5^{\circ},-10^{\circ},-15^{\circ},-20^{\circ}\right.$ forward; and $+5^{\circ},+10^{\circ},+15^{\circ},+20^{\circ}$ backward relative to the gravity) were manipulated in the present experiment. For each body orientation, 18 visual stimuli were presented to the subjects in a pseudo- 
randomised order for less than 2 min of body orientation exposure. The visual stimuli simulated a forward self-motion with upward or downward directional biases relative to the true earth horizontal direction $\left(+27^{\circ},+20^{\circ},+16^{\circ},+12.5^{\circ},+10^{\circ},+7.5^{\circ},+5^{\circ},+2.5^{\circ},+1^{\circ}\right.$ upward biases; and $-27^{\circ},-20^{\circ},-16^{\circ},-12.5^{\circ},-10^{\circ},-7.5^{\circ},-5^{\circ}$, $-2.5^{\circ},-1^{\circ}$ downward biases). For a given body orientation, each visual stimulus was repeated three times in a pseudo-randomised order. This order was strictly counterbalanced for half of the subjects. Finally, the total number of judgments was $486(3 \times 18 \times 9)$ for a total session duration of $60 \mathrm{~min}$. During the experiment, subjects were neither informed about the number and angular values of body orientation nor about the true direction of the presented visual stimuli. The instructions were frequently repeated to keep subjects alert and concentrated on the task throughout the experiment.

Data processing was first carried out on the judgment settings converted into binary values. For each simulated direction of selfmotion, a score of 1 was attributed when subjects responded they felt translating upward relative to the earth horizontal direction. Conversely, a score of 0 was attributed when subjects responded they felt translating downward relative to the earth horizontal direction. Then, a subsequent "Probit" model, using a non-linear regression analysis for dichotomic variables, was achieved on the binary responses obtained for each body orientation in order to determine the probability $p$ that subjects estimated at $50 \%$ they felt translating upward relative to the true earth horizontal direction (supplementary materials). This permitted to determine mathematically an indirect variable corresponding to the angular value of a simulated direction of self-motion at which subjects would indistinguishably feel translating forward or upward relative to the true earth horizontal direction. This specific angular value obtained for each body orientation corresponded to the subjective earth horizontal direction of self-motion and was expressed in terms of angular deviation (or error) relative to the true earth horizontal direction of self-motion. Positive angular values meant that the subjective earth horizontal direction of self-motion was estimated above the true earth horizontal direction; whereas negative angular values meant that the subjective earth horizontal direction of selfmotion was estimated below the true earth horizontal direction.

In order to test the discrimination sensitivity of the so-called subjective earth horizontal direction of self-motion relative to the chosen increments, a preliminary data analysis was conducted on the slopes of the tangent at the inflection point of the Probit function curve for each body orientation. The slopes of the tangent at the inflection point provided an indication about the sharpness relative to the transition of the response probability. A nine body orientations $\left(0^{\circ}, \pm 5^{\circ}, \pm 10^{\circ}, \pm 15^{\circ}, \pm 20^{\circ}\right)$ analysis of variance (ANOVA) with repeated measures showed no significant difference in the discrimination sensitivity whatever the body orientation $\left[F_{(3.5,38)}=0.99\right.$; Epsilon $=0.43 ; p=.42$; when corrected for sphericity with Greenhouse-Geisser epsilon adjustments; $\eta_{p}^{2}=$ $0.08 ;(1-\beta)=0.26]$.

In order to test any differences between body orientations on the subjective earth horizontal direction of self-motion, a nine body orientations $\left(0^{\circ}, \pm 5^{\circ}, \pm 10^{\circ}, \pm 15^{\circ}, \pm 20^{\circ}\right)$ ANOVA with repeated measures was then performed on the angular values of the subjective earth horizontal direction of self-motion obtained for the different body orientations. The analysis showed a significant effect of body orientation on the subjective estimates $\left[F_{(2.8,30.8)}=58.05\right.$; Epsilon $=0.35, p<.001$; when corrected for sphericity with Greenhouse-Geisser epsilon adjustments; $\eta_{p}^{2}=$ $0.84 ;(1-\beta)=1]$. Specifically, the subjective estimates ranged from $-11.67^{\circ}$ downward at $-20^{\circ}$ of body tilt to $4.25^{\circ}$ upward at $+20^{\circ}$ of body tilt relative to a true horizontal direction of self-motion. Post hoc analyses (Newman-Keuls test) confirmed the presence of significant differences between body orientations (Table 1).
Table 1

Summary table of the Newman-Keuls pairwise comparisons for the mean estimates of the subjective earth horizontal direction of self-motion The results showed significant differences between the different body orientations $\left({ }^{* * *} p<001 ;{ }^{* *} p<.01\right.$; ${ }^{*} p<.05$; ns = no significant difference)

\begin{tabular}{rlllllllll}
\hline & 20 & 15 & 10 & 5 & 0 & -5 & -10 & -15 & -20 \\
\hline 20 & - & $*$ & $* * *$ & $* * *$ & $* * *$ & $* * *$ & $* * *$ & $* * *$ & $* * *$ \\
15 & & - & $\mathrm{ns}$ & $* *$ & $* * *$ & $* * *$ & $* * *$ & $* * *$ & $* * *$ \\
10 & & & - & $\mathrm{ns}$ & $* *$ & $* * *$ & $* * *$ & $* * *$ & $* * *$ \\
5 & & & & - & $\mathrm{ns}$ & $* * *$ & $* * *$ & $* * *$ & $* * *$ \\
0 & & & & & - & $* *$ & $* * *$ & $* * *$ & $* * *$ \\
-5 & & & & & & - & $\mathrm{ns}$ & $* *$ & $* * *$ \\
-10 & & & & & & & - & $\mathrm{ns}$ & $* * *$ \\
-15 & & & & & & & & - & $*$ \\
-20 & & & & & & & & & - \\
\hline
\end{tabular}

A linear regression analysis applied to the mean estimates of the subjective earth horizontal direction of self-motion obtained for all the subjects at each body orientation yielded a significant effect of body orientation on the perceptual estimates $\left(F_{(1,7)}=3119.93\right.$; $\left.p<.001 ; R^{2}=1\right)$. The data showed that body orientation proportionally affected the error magnitude in the reported perceived direction of self-motion. Furthermore, the mean estimates of the subjective earth horizontal direction of self-motion were systematically deviated in the direction of body tilt. Specifically, when referred to no-tilt condition (which presented an offset about $-3.57^{\circ}$ below the true earth horizontal direction of self-motion), the subjective earth horizontal direction of self-motion was found lowered with forward body tilts and elevated with backward body tilts (Fig. 2). The equation of the regression function was

$$
(\zeta=0.40 \theta-3.57)
$$

where 0.40 corresponds to the gain of body orientation influence " $\theta$ " on the perceptual estimates " $\zeta$ ", with an offset of $-3.57^{\circ}$ characterizing the general lowering of the subjective estimates relative to the true earth horizontal direction of self-motion. Interestingly, numerous studies previously reported a lowering of the subjective horizontal reference for earth-based judgments given by erect observers [4,7,14,22]. For instance, Bringoux et al. [5] measured an offset on the subjective horizon in darkness which is $3^{\circ}$ lower than the true earth horizontal reference. This phenomenon may be related to the $30^{\circ}$ backward orientation of the saccular and utricular maculae relative to the naso-occipital axis of the head [19].

To our knowledge, this study represents the first attempt to evaluate the influence of body orientation on the perception of self-

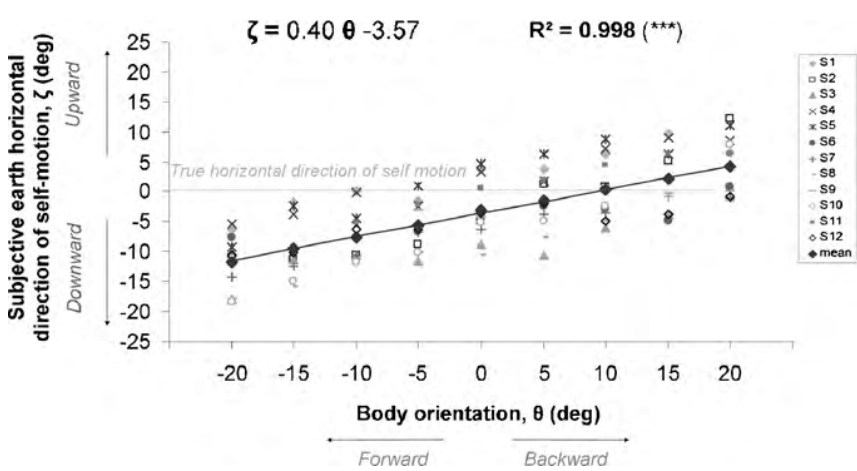

Fig. 2. Linear regression analysis applied to the mean estimates of the subjective earth horizontal direction of self-motion. Regarding estimates obtained for each of the 12 subjects in each body orientation, this figure suggests that comparable regression lines could be applied for individual profiles. The equation of the regression line shows an influence of body orientation about $40 \%$ on the subjective estimates. The coefficient -3.57 shows a general lowering of the subjective horizontal direction of self-motion relative to the true horizontal direction of self-motion at $0^{\circ}$ of pitch body orientation. The $R^{2}$ provides a measure of how well the recorded data are likely to be predicted by the linear statistical model $\left({ }^{* * *} p<.001\right)$. 
motion direction in vertical median plane, induced by optic flow. Overall, these findings clearly showed that body orientation in the sagittal plane proportionally influenced the subjective orientation of self-motion from optic flow, when judgments must be achieved relative to an earth-based reference. Specifically, systematic perceptual deviations were observed in the direction of body tilt. A first explanation may be based on the underestimation of the body tilt magnitude in space [25]. According to this interpretation, the perceptual errors may be related to a decreased sensibility of the graviceptive sensory system (mainly conveyed by otholith organs and somatosensory inputs) when the body is no more aligned with gravity, leading to a lesser sensation of tilt [12,21]. However, this hypothesis was inconsistent with other studies showing no relationship between the estimated body orientation and the perception of earth-based Refs. [7,15,16,24]. Indeed, erect subjects who were tilted in roll until they reached their perceived horizontal body orientation, did no more perceive as earth horizontal a luminous line aligned along their longitudinal body axis [15]. Thus, even if subjects were quite good at estimating their horizontal body orientation in space, they made large systematic errors in visually judging earth horizontal and vertical orientations.

A second explanation can be found in the nature of the task required. In the present experiment, estimating the perceived upward and downward direction of a forward self-motion from optic flow must be judged relative to an earth-based reference. While the visual system can determine the horizon information in presence of a structured visual environment by extrapolating from converging lines in the projection plane $[10,20,29]$, the subjective horizon (i.e., the perceived plane passing through the eye normal to the gravity) requires to be internalized under poor reliable visual condition. In this context, body orientation was shown to linearly influence the subjective horizon in a range of $\pm 20^{\circ}$ body tilt $[4,5]$. Specifically, estimates were lowered by forward tilts and elevated with backward tilts. This phenomenon was found to have important repercussions on numbers of spatial tasks relying on the subjective horizon such as perceiving the elevation of objects and estimating the possibility of passing under obstacles [3,5]. The estimation of the relative size of objects (referred on the horizon $[2,20]$ ), and the judgment of distances (based on the angular declination below the horizon [17], that is, the visual angle between eye level and object location) were also shown to be affected. Because the visual horizon constitutes the underlying reference for estimating the visually induced upward and downward direction of self-motion, we assume that a similar phenomenon would occur in the present task.

Specifically, our study clearly shows a substantial influence of a body-related reference frame on the earth-based reference frame normally required to perform the task. This phenomenon, in line with the "idiotropic vector" hypothesis given by Mittelstaedt [16] to explain the deviation of the subjective vertical toward the longitudinal body axis, was also defined in term of "egocentric attraction" induced by body tilt [3-5]. This strongly supports the existence of an interaction process between reference frames $[3,5,6]$. Specifically, regarding the slope of the regression line, directional estimates of self-motion may be performed into a composite reference frame [13], that is an intermediate state between a pure egocentric and a pure geocentric reference frame. Luyat et al. [13] evoked a similar interpretation for the mapping of the oblique effect during haptic and visual tasks of reproduction of orientation. With a slope about 1 , the subjective earth horizontal direction of self-motion would be fully oriented toward a body-related direction (e.g., the transverse plane of the head passing through the eyes [22]). In that case, the weight attributed to the egocentric reference frame would be about $100 \%$ on the visual estimates. Conversely, a slope of 0 would indicate no influence of body orientation, whereas the weight attributed to the geocentric reference frame would be con- sequently of $100 \%$. In the present study, we found an interaction between the egocentric and geocentric reference frames with a constant weight of about $40 \%$ for the egocentric reference frame. Interestingly, according to a previous study of Bringoux et al. [5] who observed a constant weight of about $20 \%$ relative to the influence of the egocentric reference frame on the same range of pitch body tilt, our results suggest that the "egocentric attraction" toward body orientation is enhanced in presence of an optic flow. Specifically, this finding could be due to the fact that a dynamic visual environment primarily specifies an egocentric direction of selfmotion [26], and may consequently increase the weight associated to the egocentric reference frame, as compared to the geocentric one.

Finally, it should be interesting to further question the influence of the required task on the present findings. Specifically, the influence of action as compared to perception only has been previously highlighted in self-motion perception [23]. Therefore, investigating the influence of body orientation in controlling the direction of self-motion may be of particular relevance in usual situations of flight including motor actions.

In conclusion, the present study provides new insights into how spatial reference frames could interact for the perception of space during visually induced self-motion. Particularly, this may be highly significant within the context of aeronautics, where fighter pilots, usually seated $30^{\circ}$ backward [18], achieve up and down earthbased judgments in poor visual conditions. Furthermore, this can also have important repercussions in helicopter of vectoring thrust vehicles' navigation, when the vehicles' longitudinal axis is not always aligned with the direction of displacement.

\section{Acknowledgements}

Aurore Bourrelly was supported by a grant from DGA-CNRS (No.2007-746). The authors are grateful to F. Buloup, A. Donneaud and C. Goulon for technical expertise, and to J. Martin-Malivel, G. Mitchell for English assistance. We also greatly thank reviewers for several helpful comments in revising the current manuscript.

\section{Appendix A. Supplementary data}

Supplementary data associated with this article can be found, in the online version, at doi:10.1016/j.neulet.2010.07.028.

\section{References}

[1] A.J. Benson, Sensory functions and limitations of the vestibular system, in: R. Warren, A.H. Wertheim (Eds.), Perception and Control of Self-motion Lawrence, Erlbaum Associates, Hillsdale, 1990, pp. 145-170.

[2] M. Bertamini, T.L. Yang, D.R. Proffit, Relative size perception at a distance is best at eye level, Percept. Psychophys. 60 (1998) 673-682.

[3] A. Bourrelly, L. Bringoux, J.L. Vercher, Influence of gaze elevation on estimating the possibility of passing under high obstacles during body tilt, Exp. Brain Res. 193 (2009) 19-28.

[4] L. Bringoux, K. Tamura, M. Faldon, M.A. Gresty, A.M. Bronstein, Influence of whole-body pitch tilt and kinesthetic cues on the perceived gravity-referenced eye level, Exp. Brain Res. 155 (2004) 385-392.

[5] L. Bringoux, G. Robic, G.M. Gauthier, J.L. Vercher, Judging beforehand the possibility of passing under obstacles without motion: the influence of egocentric and geocentric frames of reference, Exp. Brain Res. 185 (2008) 673-680.

[6] L. Bringoux, C. Bourdin, J.C. Lepecq, P. Sandor, J.M. Pergandi, D. Mestre, Interaction between reference frames during subjective vertical estimates in a tilted immersive virtual environment, Perception 38 (2009) 1053-1071.

[7] H. Ceyte, C. Cian, M. Trousselard, P.A. Barraud, Influence of perceived egocentric coordinates on the subjective visual vertical, Neurosci. Lett. 462 (2009) 85-88.

[8] J.A. Crowell, M.S. Bancks, V. Shenoy, R.A. Andersen, Visual self-motion perception during head turns, Nat. Neurosci. 1 (1998) 732-737.

[9] J.E. Cutting, Perception with An Eye for Motion, MIT Press, 1986.

[10] J.J. Gibson, The Perception of the Visual World, Houghton Mifflin, Boston, 1950

[11] J.M. Goldberg, C. Fernandez, Conduction times and background discharge of vestibular afferents, Brain Res. 122 (1977) 545-550.

[12] S. Lechner-Steinleitner, Interaction of labyrinthine and somatoreceptor inputs as determinants of the subjective vertical, Psychol. Res. 40 (1978) 65-76. 
[13] M. Luyat, S. Mobarek, C. Leconte, E. Gentaz, The plasticity of gravitational reference frame and the subjective vertical: peripheral visual information affects the oblique effect, Neurosci. Lett. 385 (2005) 215-219.

[14] R. MacDougall, The subjective horizon, Psychol. Rev. 4 (1903) 145-166.

[15] F. Mast, T. Jarchow, Perceived body position and the visual horizontal, Brain Res. Bull. 40 (1996) 393-398.

[16] H. Mittelstaedt, Somatic graviception, Biol. Psychol. 42 (1996) 53-74.

[17] T.L. Ooi, B. Wu, Z.J. He, Distance determined by the angular declination below the horizon, Nature 414 (2001) 197-200.

[18] C. Roumes, J.Y. Grau, Rafale: a human factors view of the man machine interface, Med. Aeronaut. Spat. 45 (2003) 23-26.

[19] U. Rosenhal, Vestibular macular mapping in man, Ann. Otol. Rhinol. Laryngol. 81 (1972) 339-351.

[20] H.A. Sedgwick, Space perception, in: K.R. Boff, L. Kaufman, J.P. Thomas (Eds.) Handbook of Perception and Human Performance, vol. 21, Willey, New York, 1986, pp. 1-21 (chapter 57).

[21] H. Shöne, On the role of gravity in human spatial orientation, Aerosp. Med. 35 (1964) 764-772.
[22] A.E. Stoper, M.M. Cohen, Effect of structured visual environments on apparent eye level, Percept. Psychophys. 46 (1989) 469-475.

[23] L. Tremblay, D. Elliott, Contribution of action to perception of self-orientation in humans, Neurosci. Lett. 349 (2003) 99-102.

[24] M. Trousselard, P.A. Barraud, V. Nougier, C. Raphel, C. Cian, Contribution of tactile and interoceptive cues to the perception of the direction of gravity, Cogn. Brain Res. 20 (2004) 355-362.

[25] A.D. van Beuzekom, J.A.M. van Gisbergen, Properties of the internal representation of gravity inferred from spatial-direction and body-tilt estimates, J. Neurophysiol. 84 (2000) 11-27.

[26] A.V. van den Berg, Judgements of heading, Vision Res. 36 (1996) 2337-2350.

[27] W.H. Warren Jr., M.W. Morris, M. Kalish, Perception of translational heading from optical flow, J. Exp. Psychol. Hum. Percept. Perform. 14 (1988) 646660.

[28] M. Wexler, Voluntary head movement and allocentric perception of space, Psychol. Sci. 14 (2003) 340-346.

[29] J. Wu, Z.J. He, T.L. Ooi, Visually perceived eye level and horizontal midline of the body trunk influenced by optic flow, Perception 34 (2005) 1045-1060. 